hours. Physiologic parameters were monitored and pain scores assessed.

Results The median fentanyl concentrations were $0.18,0.15$, 0.15 and $0.57,0.37,0.35 \mathrm{ng} / \mathrm{mL}$ at $15-31$ minutes, two and four hours and the half-lives were 1.6 to 20.5 or 4.1 to 32.6 hours for the low and high dose groups, respectively. A significant correlation was seen between weight at study inclusion and half-life (Spearman's $r=-0.9, \mathrm{p}<0.001$ ), volume of distribution $(r=-0.8, \mathrm{p}<0.01)$ and clearance $(r=-0.9, \mathrm{p}<$ $0.01)$ in the low dose group $(\mathrm{n}=9)$. Pain assessment results were not correlated to pharmacokinetic variables. Fentanyl was well tolerated.

Conclusion The inter-individual variation of fentanyl pharmacokinetics is large in preterm infants and the dose of $0.5 \mathrm{mg} /$ $\mathrm{kg}$ seems too small for skin-breaking procedures.

Disclosure(s) Nothing to disclose

\section{P51 A COMBINED EXPERIMENTAL APPROACH TO ASSESS INTESTINAL DRUG ABSORPTION IN EARLY CHILDHOOD}

${ }^{1} \mathrm{M}$ Kiss* ${ }^{2}{ }^{2} \mathrm{~J}$ Nicolaï, ${ }^{1} \mathrm{~J} \mathrm{MMW}$ van den Heuvel, ${ }^{3} \mathrm{R}$ Mbasu, ${ }^{4} \mathrm{SM}$ Botden, ${ }^{1} \mathrm{JB}$ Koenderink, ${ }^{1}$ FGM Russel, ${ }^{2} \mathrm{~A}-\mathrm{L}$ Ungell, ${ }^{1,5} \mathrm{SN}$ de Wildt. 'Department of Pharmacology and Toxicology, Radboud University Medical Center, Nijmegen, The Netherlands; ${ }^{2}$ Development Science, UCB Biopharma SPRL, Braine-l'Alleud, Belgium; '3evelopment Science, UCB Celltech, Slough, UK; ${ }^{4}$ Department of Surgery-Division of Pediatric Surgery, Radboud UMC-Amalia Children's Hospital, Nijmegen; ${ }^{5}$ Intensive Care and Department of Pediatric Surgery, ErasmusMC-Sophia Children's Hospital, Rotterdam, The Netherlands

\subsection{6/archdischild-2019-esdppp.89}

Background Drug transporters and metabolizing enzymes located in the epithelial lining of the intestine limit or enhance systemic drug exposure. During child development, the abundance and activity of these transporters and enzymes - determining how fast and how much of a drug is being absorbed into the circulation - changes from birth to adolescence. As most drugs given to children are taken by mouth, the aim of this project is to study the abundance and activity of transporters and metabolizing enzymes, involved in the intestinal absorption of drugs.

Methods The ex vivo Ussing chamber with pediatric small intestinal tissue is applied to evaluate intestinal drug absorption and metabolism. ${ }^{1}$ Transport and metabolism of a selection of drug molecules is assessed across these tissues by sampling the donor and receiver compartment at different intervals and sample analysis by LC-MS/MS. Viability, functionality and integrity of the tissues are monitored using electrophysiological parameters (dP, R, I). Ussing chamber experiments are combined with a targeted proteomics approach to quantify drug transporter and metabolizing enzyme abundance in these tissues.

Results An Using chamber method has been successfully set up using both adult and pediatric intestinal tissue. To date samples from three children of different ages have been evaluated and show promising results. Tissue from the same patients has been stored for proteomics analysis.

Conclusion The Ussing method presents an innovative, feasible approach to study active intestinal transport in children. Further studies are now underway to elucidate age-related variation in intestinal transport and metabolism.

\section{REFERENCE}

1. Sjöberg $\AA$, Lutz M, Tannergren C, Wingolf C, Borde A, Ungell AL:Comprehensive study on regional human intestinal permeability and prediction of fraction absorbed of drugs using the Ussing chamber technique. Eur J Pharm Sci 2013 Jan 23:48(1-2):166-80.

Disclosure(s) The collaboration project is financed by the Ministry of Economic Affairs by means of the PPP Allowance made available by the Top Sector Life Sciences \& Health to stimulate public-private partnerships. Johan Nicolaï, Richard Mbasu and Anna-Lena Ungell are employees of UCB Biopharma SPRL.

\section{P52 LOW AND ERRATIC EXPOSURE OF ORAL ACETAMINOPHEN IN CRITICALLY ILL CHILDREN DETERMINED WITH A ${ }^{14}$ C MICROTRACER STUDY: A CASE FOR IV ACETAMINOPHEN FOR ACUTE PAIN MANAGEMENT?}

${ }^{1} \mathrm{~N}$ Kleiber*, ${ }^{2} \mathrm{E}$ Calvier, ${ }^{3} \mathrm{MG}$ Mooij, ${ }^{2}$ EHJ Krekels, ${ }^{4} \mathrm{WH}$ Vaes, ${ }^{5} \mathrm{AD}$ Windhorst, ${ }^{6} \mathrm{H}$ Hendrikse, ${ }^{3} \mathrm{D}$ Tibboel, ${ }^{2} \mathrm{CAJ}$ Knibbe, ${ }^{7} \mathrm{SN}$ de Wildt. ${ }^{1}$ Pediatrics and Clinical Pharmacology Unit, $\mathrm{CHU}$ Sainte-Justine, Montréal, QC, Canada; ' ${ }^{2}$ eiden Academic Centre for Drug Research, Leiden University, Leiden; ${ }^{3}$ Erasmus MC - Sophia Childrens Hospital, Rotterdam; ${ }^{4}$ TNO, Zeist; ${ }^{5}$ Amsterdam University Medical Center; ${ }^{6}$ Vrije Universiteit Amsterdam (VU), Amsterdam; ${ }^{7}$ Department of Pharmacology and Toxicology, Radboud University Medical Center, Nijmegen, The Netherlands

\subsection{6/archdischild-2019-esdppp.90}

Background Despite being the most commonly used analgesic and antipyretic, oral APAP bioavailability has not been determined in children.

The aim of this study is to compare exposure after oral vs iv APAP using the PK data of the first pediatric oral bioavailability $14 \mathrm{C}$ microtracer study.

Methods

Design bioavailability microtracer population PK study

Participants patients $<6$ yrs old in the pediatric ICU who received $15 \mathrm{mg} / \mathrm{kg}$ iv APAP $\mathrm{q} 6 \mathrm{~h}$

Intervention a single microdose of ${ }^{14 \mathrm{C}} \mathrm{APAP}(3 \mu \mathrm{g} / \mathrm{kg})$ given orally at the same time as a therapeutic iv dose

Data collection Blood was sampled 8 times up to 24 h postdose

Data analysis population PK analysis using NONMEM. Based on the model, exposure after oral vs iv was compared by simulating the concentration-time profiles and Css (targeted: 10 $\mathrm{mg} / \mathrm{L} \pm 20 \%$ deviation).

3 doses were simulated: $15 \mathrm{mg} / \mathrm{kg} \mathrm{q} 6 \mathrm{~h}$ oral and iv and $22.5 \mathrm{mg} / \mathrm{kg}$ oral q6h. 1000 simulations were performed and the percentage of patients reaching the targeted mean Css of $10 \mathrm{mg} / \mathrm{L} \pm 20 \%$ were compared.

Results Oral bioavailibity was 72\% (range:11-91\%). After 15 $\mathrm{mg} / \mathrm{kg}$ APAP, the median simulated oral Css was subtherapeutic (6.5 $\mathrm{mg} / \mathrm{L})$, but therapeutic $(10 \mathrm{mg} / \mathrm{L})$ for IV dosing $(15 \mathrm{mg} /$ $\mathrm{kg}$ ). Patients were 2.5 times less likely to reach therapeutic plasma concentrations with $15 \mathrm{mg} / \mathrm{kg}$ oral vs iv APAP.

With the maximal recommended oral doses of $22.5 \mathrm{mg} / \mathrm{kg}$ $6 \mathrm{~h}$ aimed to overcome the $72 \%$ bioavailability, median mean Css were therapeutic but overexposure and underexposure were more common than with iv (37 vs $32 \%$ Css $<8 \mathrm{mg} / \mathrm{L}$ and 30 vs $21 \%$ Css $>12 \mathrm{mg} / \mathrm{L}$ ).

Conclusion Compared to IV, the usual $(15 \mathrm{mg} / \mathrm{kg})$ oral APAP doses result in low systemic exposure with subsequent risk of therapeutic failure. When oral doses are increased to overcome the low bioavailability, underdosing still occurs and overdosing was observed in patients with high bioavailability. IV 
APAP should therefore be preferred for acute pain management.

Disclosure(s) Nothing to disclose

\section{P53 EXPOSURE OF INFANTS TO BROMINATED FLAME RETARDANTS THROUGH BREAST-MILK}

${ }^{1} \mathrm{E}$ Kohn ${ }^{*}{ }^{2} \mathrm{~S}$ Efreim, ${ }^{3,4} \mathrm{R}$ Lubetzky, ${ }^{3,4} \mathrm{D}$ Mandel, ${ }^{3,4} \mathrm{R}$ Marom, ${ }^{4,5} \mathrm{M}$ Betser, ${ }^{4,6} \mathrm{R}$ Keidar, ${ }^{4,6} \mathrm{~A}$ Livne, ${ }^{7} \mathrm{~A}$ Levy, ${ }^{8} \mathrm{P}$ Factor-Litvak, ${ }^{2} \mathrm{~S}$ Soback, ${ }^{2} \mathrm{M}$ Britzi, ${ }^{1,4} \mathrm{M}$ Berkovitch. ${ }^{1}$ Clinical Pharmacology and Toxicology, Assaf Harofeh Medical Centre, Zerifin; ${ }^{2}$ Residues Lab, Kimron Veterinary Institute, Beit-Dagan; ${ }^{3}$ Departments of Neonatology and Pediatrics, Dana Childrens' Hospital, Tel-Aviv Medical Centre; ${ }^{4}$ Sackler Faculty of Medicine, Tel-Aviv University, Tel Aviv; ${ }^{5}$ Obstetrics and Gynecology Division; ${ }^{6}$ Department of Neonatology, Assaf Harofeh Medical Centre, Zerifin; ${ }^{7}$ Department of Public Health, Ben Gurion University of the Negev, Beer Sheva, Israel; ${ }^{8}$ Department of Epidemiology, Mailman School of Public Health, Columbia University, New York, NY, USA

\subsection{6/archdischild-2019-esdppp.91}

Introduction Polybrominated Diphenyl Ethers (PBDEs) are non-biodegradable flame retardants, accumulated in biological systems and acting as endocrine disruptors. Breast feeding is a major route of exposure in infancy. Taken together with the critical development of this age and the potential adverse effects of PBDEs, it is important to monitor these contaminants in breastmilk.

Objective To evaluate the exposure of infants to PBDEs

Methods 343 families were recruited during 2013-2016 in Assaf Harofeh and Ichilov to create the AHI-EHF cohort. Maternal blood and urine, cord blood, breast milk and meconium were collected. Participants filled out questionnaires about socio-demographic status, medical history, exposures and life habits. Colostrum samples were collected from women at the maternity department. PBDEs in colostrum and Infant formulas levels were analyzed using GC-MS

Results and discussion Out of 183 serum samples, only 11 (6\%) detectable levels of PBDEs. PBDEs were found in all colostrum samples. The average concentration of total PBDEs in breastmilk was $714 \mathrm{ng} / \mathrm{L}$. PBDEs levels were also measured in three infant formulas. Unlike breastmilk, infant formulas had of only 3 congeners and levels were relatively low. The average concentration of total PBDEs in infant formulas was $153 \mathrm{ng} / \mathrm{L}$. PBDEs, were found to be negatively correlated to anno-penile index (API) which serve as a marker for endocrine disruption.

Conclusions PBDEs levels in breast milk are higher than levels in some European countries, but lower than in North America. PBDEs might have negative influence on AGD in boys. Maternal exposure to PBDEs and the significance of it should be further investigated.

Disclosure(s) Nothing to disclose

\section{P54 KIDS-STEP: A SWISS MULTI-CENTRE RCT ON EFFECTIVENESS OF ADJUNCT BETAMETHASONE THERAPY IN HOSPITALISED CHILDREN WITH COMMUNITY ACQUIRED PNEUMONIA}

\footnotetext{
${ }^{1} \mathrm{M}$ Kohns Vasconcelos*, ${ }^{1} \mathrm{R}$ Santoro, ${ }^{2} \mathrm{M}$ Coslovsky, ${ }^{1} \mathrm{~J}$ van den Anker, ${ }^{1} \mathrm{JA}$ Bielicki. ${ }^{1}$ Department for Paediatric Pharmacology, University Childrens' Hospital Basel (UKBB); ${ }^{2}$ Department of Clinical Research, Clinical Trial Unit, University and University Hospital of Basel, Basel, Switzerland
}

10.1136/archdischild-2019-esdppp.92
Background The incidence of community-acquired pneumonia (CAP) in young children is high (20-30/1000 child-years) and is associated with a high rate of hospitalisation (around 10/ 1000 child-years). In adults, a benefit of adjunct corticosteroids on time to clinical stability and hospital discharge has been observed and confirmed in systematic reviews and metaanalyses. In contrast, only few small trials have addressed the potential impact of oral steroid treatment in CAP during childhood. The purpose of this study is to concurrently evaluate whether adjunct treatment with corticosteroids in children hospitalised with CAP is more effective in terms of the proportion of children reaching clinical stability and whether such adjunct treatment is no worse in terms of CAP relapse.

Methods Children in KIDS-STEP ${ }^{1}$ receive either oral betamethasone or oral placebo dosed once daily for two consecutive days. We include 700 children from age 1 weighing at least 7 kilograms and up to a body weight of 35 kilograms and age below 10 years hospitalised for CAP using a clinical diagnosis. Co-primary outcomes are (a) The proportion of children clinically stable at 48 hours after randomisation. (b) The proportion of children with CAP-related readmission within 28 days after randomization. Secondary outcomes will be captured to further evaluate the efficacy and safety of adjunct oral steroids in the management of childhood CAP, including proportion of children experiencing solicited side effects of the trial treatment and/or serious adverse events, time to hospital discharge after index hospitalisation in days, time away from routine child care and away from work (for parents) in days up to 28 days after randomisation and total antibiotic exposure in days up to 28 days after randomisation.

Results Enrolment started in November 2018 and is currently proceeding at approximately 1 participant per participating hospital per week.

\section{REFERENCES}

1. Study registration: BASEC - EKNZ 2018-00563

Disclosure(s) Nothing to disclose

\section{P55 PREVENTING INAPPROPRIATE HYDROXYUREA DOSING IN CHILDREN BY INTRODUCING A CHILD-APPROPRIATE PREPARATION}

G Koren*. Maccabi Research Institute, Tel Aviv, Israel

\subsection{6/archdischild-2019-esdppp.93}

Background Hydroxyurea (HU) is the only FDA- approved disease- modifying drug for sickle cell disease, by inducing the production of fetal hemoglobin and thus decreasing the sickling of red blood cells. Till recently HU was available only in adult doses of $1000 \mathrm{mg}$. This meant that to aim at the standard dose of $20 \mathrm{mg} / \mathrm{kg} / \mathrm{d}$, most young children had to be overdosed, or the dose had to be fluctuated daily to achieve the aimed mean dose. Because adherence improves with unchanged daily dose, and due to the more than 10 fold variability in HU pharmacokinetics in children, there was an urgent need for a pediatric formulation of HU.

Methods and results This issue has been solved with the FDA approval of the French-originated orphan HU, Siklos, a preparation of 50 and $100 \mathrm{mg}$, which prevents the risk of inappropriate dosing in children.

Studies show that the child appropriate dose preparation much more closely allow young children to receive 